\title{
Plantar forefoot pain: ultrasound findings before and after treatment with custom-made foot orthoses
}

\author{
Domenico Albano ${ }^{1,2,3}$ (C) Carlo Bonifacini ${ }^{4} \cdot$ Stefania Zannoni ${ }^{5}$. Susan Bernareggi ${ }^{1} \cdot$ Carmelo Messina $^{1}$. \\ Massimo Galia ${ }^{2} \cdot$ Luca Maria Sconfienza ${ }^{1,6}$
}

Received: 15 December 2020 / Accepted: 30 March 2021 / Published online: 21 April 2021

(c) The Author(s) 2021

\begin{abstract}
Purpose No prior studies investigated the role of ultrasound in the assessment of response of patients undergoing treatment of metatarsalgia with custom-made orthoses. Our aim was to describe ultrasound findings of patients with plantar forefoot pain treated with custom-made foot orthoses.

Methods Twenty patients (15 females; mean age: $62.6 \pm 11$ years) affected by metatarsalgia in 27/40 feet underwent clinical evaluation before, three months and six months after treatment with custom-made full foot insole with a support proximal and an excavation below the painful metatarsals. Ultrasound was performed before and three months after the use of orthoses to examine the presence of intermetatarsal/submetatarsal bursitis, metatarsophalangeal joints effusion, anterior plantar fat pad oedema, flexor tendinitis/tenosynovitis, and Morton's neuroma. Outcome measures were clinical response with Foot Function Index (FFI)/Visual Analogue Scale (VAS) and ultrasound features changes.

Results Median VAS and FFI before treatment were 8[5-8.5] and 45.85[32.4-59.4], respectively. After 3 and 6 months of insoles use, both median VAS (2.5 [0-5] and 0 [0-2.75], respectively) and median FFI (7.9 [3.95-20] and 0 [0-3.95], respectively) showed a significant reduction in pain and disability $(\mathrm{p}<.001)$. Before treatment, ultrasound revealed 22 intermetatarsal bursitis, 16 submetatarsal bursitis, 10 joint effusions, 20 fat pad oedema, 3 flexor tendinitis/tenosynovitis and 3 Morton's neuromas. After 3 months of treatment, a significant decrease of intermetatarsal bursitis $(7, p<.001)$ was observed. No significant changes were observed in any other ultrasound parameters.

Conclusion Ultrasound might be able to detect some imaging features associated with the response of forefoot pain to custom-made foot orthoses, especially intermetatarsal bursitis.
\end{abstract}

Keywords Metatarsalgia $\cdot$ Bursitis $\cdot$ Morton's neuroma $\cdot$ Ultrasound $\cdot$ Orthoses

Domenico Albano and Carlo Bonifacini share the first author position as they equally contributed to this work.

Domenico Albano

domenico.albano@unipa.it

1 IRCCS Istituto Ortopedico Galeazzi, Milano, Italy

2 Sezione di Scienze Radiologiche, Dipartimento di Biomedicina, Neuroscienze e Diagnostica Avanzata, Università di Palermo, Palermo, Italy

3 Italian Society of Medical and Interventional Radiology (SIRM), SIRM Foundation, 20122 Milan, Italy

4 Foot and Ankle Surgery, IRCCS Istituto Ortopedico Galeazzi, Milan, Italy

5 Scuola di Specializzazione in Radiodiagnostica, Università Degli Studi di Milano, Milan, Italy

6 Dipartimento di Scienze Biomediche Per la Salute, Università Degli Studi di Milano, Milano, Italy

\section{Introduction}

Metatarsalgia is one of the most frequent painful conditions of the forefoot [1]. Patients usually present with forefoot plantar pain during weight-bearing activities, typically in the area across the second through the fourth metatarsal heads. Metatarsalgia typically develops secondary to mechanical disorders that lead to high-impact loading under the metatarsal heads, but it can also originate from trauma, instability of the metatarsophalangeal joints, inflammatory or infectious processes, and bone tumours $[2,3]$. Metatarsalgia may be treated either conservatively (use of metatarsal pads or bars, physical therapy, steroid or alcohol injection, radiofrequency ablation) $[4,5]$ or surgically $[6,7]$, according to its main cause. The first step is generally appropriate footwear with custom-made 
or prefabricated insoles to reduce plantar pressure and subsequent pain in the metatarsal head region [7, 8]. Insoles have been shown to reduce post-fatigue loading under the toes and the midfoot, with custom-made insoles being able to further decrease loading under the heel during running when compared with prefabricated insoles [8]. Weight-bearing radiographs, ultrasound, and magnetic resonance imaging can all be used to reach the diagnosis to guide treatment [9-18]. Foot and ankle ultrasound has been increasingly used as a diagnostic tool with the ability to dynamically evaluate during motion and direct palpation [19,22]. Most of the causes of metatarsalgia can be diagnosed by forefoot ultrasound, including bursitis, Morton's neuroma (MN), tendinitis/tenosynovitis, and neoplastic masses. According to the last clinical guidelines for musculoskeletal ultrasound by the European Society of Musculoskeletal Radiology, ultrasound is recommended as the first-choice imaging modality for both ankle/foot tendinopathies and MN [23, 24]. However, no previous studies have investigated how ultrasound features change over time after conservative treatment of metatarsalgia. Thus, the aim of this study was to describe ultrasound findings of patients with plantar forefoot pain treated with custom-made foot orthoses.

\section{Materials and methods}

\section{Study design}

This retrospective study evaluated ultrasound findings of patients with metatarsalgia managed at the Podiatry Clinic, the Unit of Diagnostic and Interventional Radiology and the Foot and Ankle Surgery department of our Institution from December 2017 to October 2018. Our study included a consecutive cohort of patients with metatarsalgia examined clinically and using ultrasound at baseline and three months after conservative treatment with a custom-made full foot insole with proximal support and excavation below the painful metatarsals. All patients were then clinically re-evaluated after 6 months as a routine clinical procedure at our Institution. We included patients $\geq 18$ years of age, with metatarsalgia of central metatarsal heads who presented for initial evaluation for localized pain in the forefoot (second, third, and fourth metatarsals and their respective metatarsophalangeal joints) made worse with weight bearing. Exclusion criteria were known autoimmune and rheumatic diseases, behavioural and mental disorders, pregnancy, recent trauma, neurodegenerative diseases, and systemic neuropathies. This study was approved by our Institutional Review Board, with a waiver for patients' informed consent.

\section{Clinical assessment and orthoses construction}

Every patient first underwent clinical assessment by both an experienced orthopedist and a podiatrist. The diagnosis of metatarsalgia was made when pain in the forefoot area with increased stress over the metatarsal head region was observed. A general clinical examination was performed to assess any possible cause of metatarsalgia. Standard clinical examination included the following steps. Initially, the foot was inspected for skin integrity, swelling, and temperature. Then, a biomechanical examination of the tibiotarsal, subastragalic, mediotarsal, Lisfranc, and metatarsophalangeal joints was performed, to evaluate the range of motion of every joint, in order to exclude the presence of joint stiffness. The presence of deformities of the toes, their mobility, and possible subluxations were analyzed. Specific attention was paid to the first ray to determine its position (neutral, dorsiflexed, or plantarflexed) and correct mobility. The medial longitudinal arch, the alignment of the hindfoot, the forefoot, and their relationship were examined. The plantar fascia was palpated to assess plantar fasciitis and the course of the Achilles tendon to assess any painful areas. To investigate the presence of bursitis, acupressure of metatarsal heads and intermetatarsal spaces was applied and the Mulder test was performed to assess the presence of a MN. Finally, the patient was asked to walk barefoot to examine the movement of the foot during the gait cycle, the correct support of the heel and forefoot, and to assess the presence of asymmetries during the gait. The Visual Analog Scale (VAS) was used to measure subjective pain experienced by the patient [25]. Foot symptoms were determined using the Foot Function Index (FFI) [26], a validated patient-administered index composed of 23 questions divided into three groups, developed to measure the impact of foot disease on its function in terms of pain, disability, and activity restriction. All patients were treated with orthotics made on a two-dimensional impression, placing on an expanded ethylene vinyl acetate material a retrocapped latex bar located $6.5 \mathrm{~mm}$ proximally to the second metatarsal head and a polypropylene support of the longitudinal medial vault (Fig. 1). Every patient was advised to wear sneakers with a heel support. No other treatments were used on these patients.

\section{Ultrasound evaluation}

Baseline and 3-months ultrasound examination was performed by the same operator, a senior radiologist with 10 years experience in musculoskeletal ultrasound. All examinations were performed with the same linear transducer (5-14 MHz, Preirus 400, Hitachi, Japan). During 


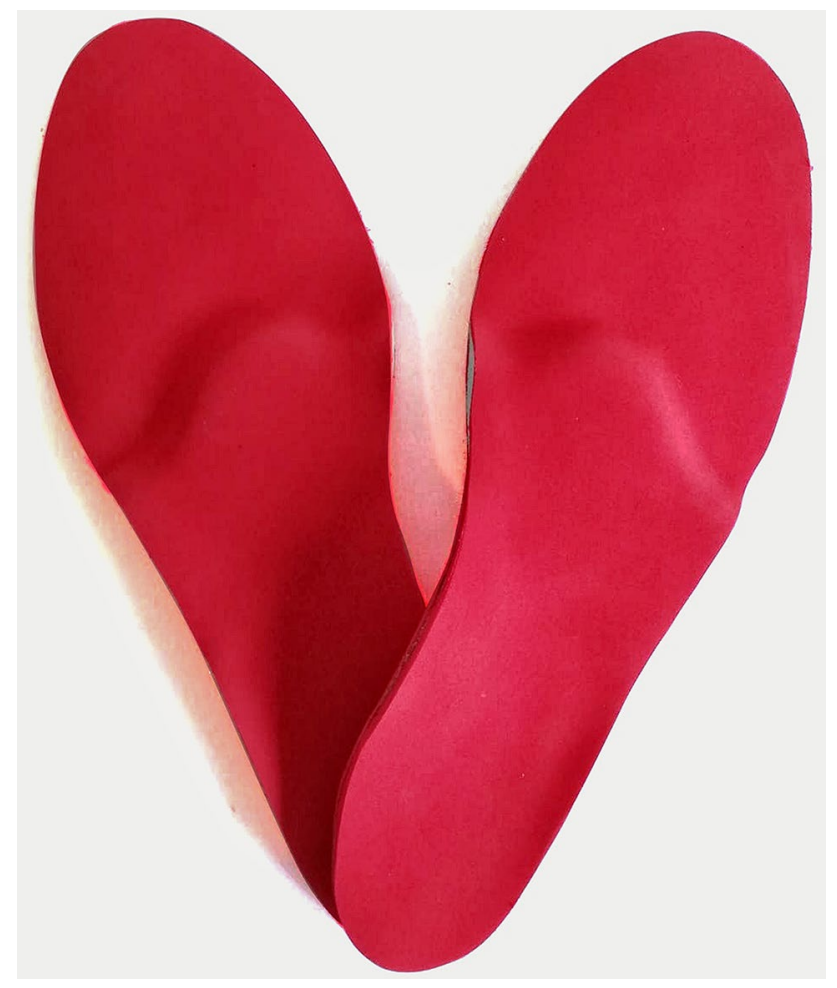

Fig. 1 The orthotics were made placing on an expanded ethylene vinyl acetate material a retrocapped latex bar at the level of the metatarsal heads and a polypropylene support of the longitudinal medial vault

the ultrasound examinations, patients were laying supine on the examination bed with the ankle relaxed. A dorsal approach was performed to identify pathological effusion in the metatarso-phalangeal joints. As a small amount of metatarsophalangeal joint effusion is physiologic, we considered this finding pathologic when an increase in intra-articular fluid was observed in at least one metatarsophalangeal joint when compared with the other ipsilateral and contralateral ones. A plantar approach was used to detect the presence of: intermetatarsal bursitis as a hypo to anechoic fluid collection in the intermetatarsal space, indeed, intermetatarsal bursa is not visible with ultrasound in normal conditions [27]; adventitious submetatarsal bursitis as a compressible hypo to anechoic fluid collection beneath the metatarsal heads [28]; MN as a round or peanut-shaped hypoechoic nodule placed in the plantar aspect of the intermetatarsal space being more visible squeezing the metatarsals during the scan [29]; flexor tendinitis as thickening/thinning of flexor tendons and flexor tenosynovitis as increased fluid content within tendons sheath [30]; oedema of the anterior plantar fat pad that has been scarcely reported in literature, although in our experience is frequently encountered in patients with metatarsalgia. As mentioned, the evaluation of the intermetatarsal spaces was dynamically performed in coronal and sagittal planes performing the Mulder manoeuvre to increase diagnostic sensitivity for MN. This evaluation was done scanning the plantar surface of the forefoot, applying pressure on the dorsal aspect of the intermetatarsal space with a finger not involved in probe holding and clasping the metatarsal heads with the fingers of the left hand [31]. The plantar fascia was also checked to exclude pathologic conditions of the fascia potentially determining metatarsalgia including plantar fasciits (as a hypoechoic appearance and thickening of more than $4 \mathrm{~mm}$ of the fascia), plantar tear (as a partial or complete disruption of plantar fibres), and plantar fibromatosis (as single or multiple eccentric and hypoechoic nodular thickenings of the fascia) [32]. Further, the ultrasound examination included the evaluation of metatarsal bony profiles to search for signs of stress fractures including cortical lines and callus formation [29].

\section{Statistical analysis}

VAS and FFI, together with ultrasound findings, were used for baseline evaluation and to monitor the response to treatment in follow-up examinations. Continuous variables are reported as mean \pm standard deviation. Discrete variables are summarized as median and interquartile range. Proportions are expressed as percentages. Frequencies were compared using the McNemar test. Paired $t$ test for parametric continuous data was also used. Statistical analysis was performed using SPSS ${ }^{\circledR}$ software (v. 25, IBM, Armonk, New York, NY). A P value lower than 0.05 was considered statistically significant [33].

\section{Results}

\section{Clinical findings}

A total of 20 patients ( 15 women, 5 men; mean age: $62.6 \pm 11$ years; range $36-78$ years) with forefoot pain in 27 out of 40 feet were included in this study, with seven of them having bilateral pain. The median VAS score and the median FFI score before treatment were 8 [interquartile range $=5-8.5]$ and 45.85 [32.4-59.4], respectively. After 3 and 6 months of insoles use, both median VAS (2.5 [0-5] and 0 [0-2.75], respectively) and median FFI (7.9 [3.95-20] and 0 [0-3.95], respectively) showed a significant reduction in pain and disability $(p<0.001)$ (Fig. 2$)$.

\section{Ultrasound findings}

Intermetatarsal bursitis was observed in $81 \%$ of feet $(22 / 27)$ at baseline ultrasound and in $25 \%(7 / 27)$ at three months, with a statistically significant reduction of this imaging 
Fig. 2 Boxplots show the progressive and significant reduction of both median VAS score and the median FFI score from baseline to 3 months (1FU) and 6 months $(2 \mathrm{FU})$ evaluation $(p<.001)$
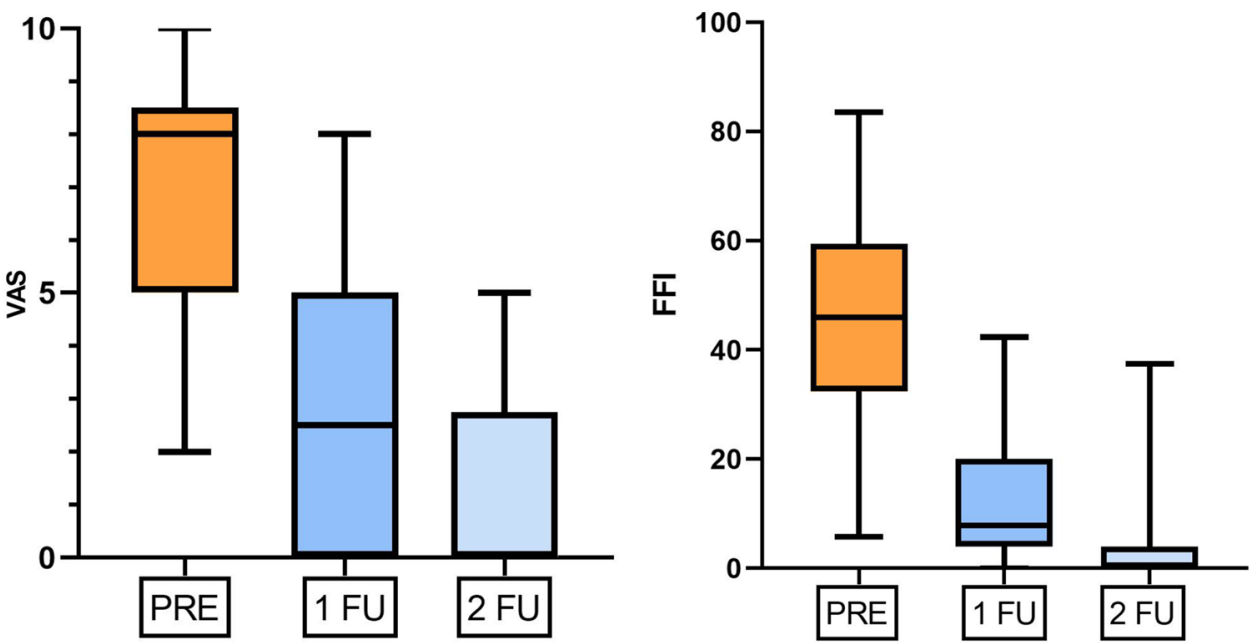

Table 1 All ultrasound findings before and after three months of conservative treatment

\begin{tabular}{lllc}
\hline & Pre-treatment & Post-treatment & $p$ value \\
\hline Intermetatarsal bursitis & $81 \%(22 / 27)$ & $25 \%(7 / 27)$ & $<0.001^{*}$ \\
Submetatarsal bursitis & $59 \%(16 / 27)$ & $37 \%(10 / 27)$ & 0.074 \\
Joint effusion & $37 \%(10 / 27)$ & $18 \%(5 / 27)$ & 0.065 \\
Fat pad imbibition & $74 \%(20 / 27)$ & $51 \%(14 / 27)$ & 0.074 \\
Morton's neuroma & $11 \%(3 / 27)$ & $11 \%(3 / 27)$ & $>0.999$ \\
Tendinitis/tenosynovitis & $11 \%(3 / 27)$ & $11 \%(3 / 27)$ & $>0.999$ \\
\hline
\end{tabular}

*Indicates a $P$ value lower than 0.05 , which was considered as statistically significant feature $(p<0.001)$. At baseline ultrasound, $59 \%$ of feet (16/27) showed submetatarsal bursitis, 37\% (10/27) joint effusion, $74 \%$ (20/27) oedema of the anterior plantar fat pad. After three months of conservative therapy, we noted a reduced frequency of these ultrasound findings, but without statistically significant differences from baseline ultrasound ( $p>0.06)$. Further, both $\mathrm{MN}$ and flexor tendinitis/ tenosynovitis were observed in $11 \%$ of feet $(3 / 27)$ at baseline ultrasound, with no improvement at 3-months sonographic examination. Neither pathologic findings of the plantar fascia nor signs of stress fractures were observed in our series. All ultrasound findings before and after three months of

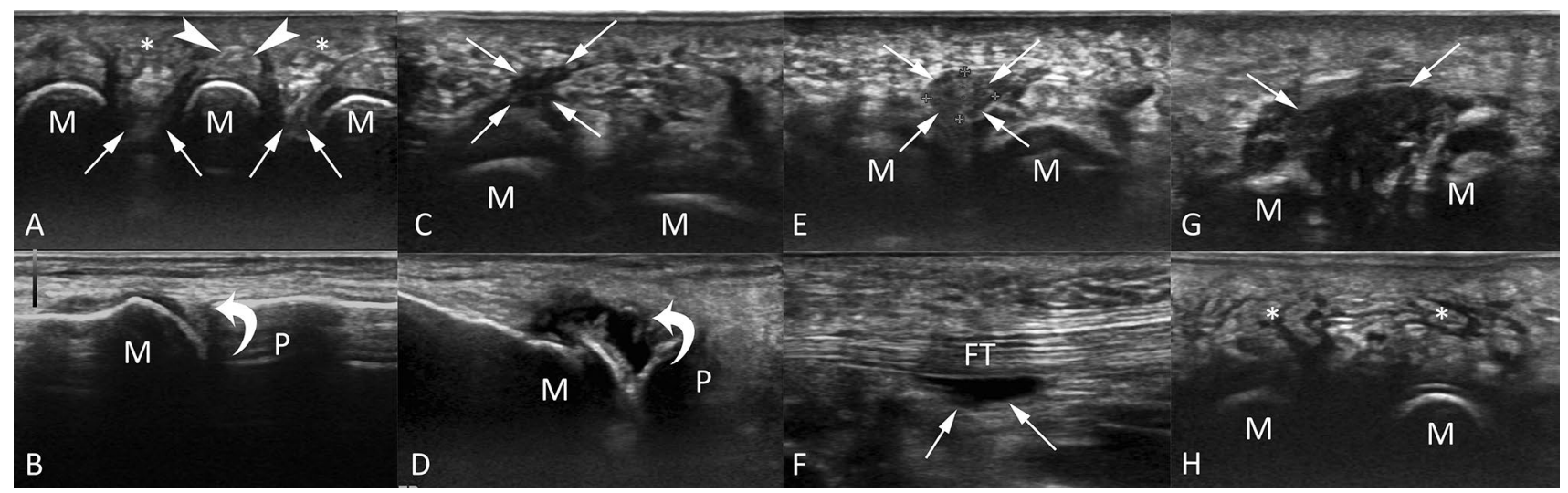

Fig. 3 Ultrasound findings of some representative cases from our study population. Transverse image with plantar approach a of a patient with normal anterior plantar fat pad (asterisks), flexor tendon (headarrows), and intermetatarsal spaces (arrows). Longitudinal image with dorsal approach $\mathbf{b}$ of the first metatarsophalangeal joint with no effusion (curved arrow). Transverse image with plantar approach of submetatarsal bursitis (c, arrows). Longitudinal image with dorsal approach $\mathbf{d}$ of the first metatarsophalangeal joint with pathological effusion (curved arrow). Transverse image with plantar approach of Morton's neuroma (e, arrows). Longitudinal image with plantar approach of flexor tendinitis (f, arrows). Transverse image with plantar approach of intermetatarsal bursitis (g, arrows). Transverse image with plantar approach $\mathbf{h}$ of a patient with oedema of the anterior plantar fat pad (asterisks). $\mathrm{M}=$ metatarsal; $\mathrm{P}=$ proximal phalange; $\mathrm{FT}=$ flexor tendon 
conservative treatment are shown in Table 1. Figure 3 shows the ultrasound findings of some representative cases from our study population.

\section{Discussion}

Our main finding was that some ultrasound features seem to be associated with the response of metatarsalgia to custommade foot orthoses, particularly the decrease of intermetatarsal bursitis. Conversely, we did not observe MN and flexor tendinitis/tenosynovitis changes after conservative treatment of forefoot pain.

The use of orthotics in patients affected by forefoot pain has a proven beneficial effect on pain relief. This is probably related to the reduction in pressure on the metatarsal heads, which invariably increases with age. Indeed, in older people, plantar fat pads show greater stiffness, dissipate more energy when compressed and are slower to recover after the load is removed. This explains the advanced mean age of patients included in our series (62.6 years) [34]. In daily practice, metatarsal head unloading is the main strategy to prevent pressure sores and to reduce forefoot pain [35]. In 2018, a systematic review stated that the use of custom-made foot orthoses improves the level of forefoot pain in patients affected by rheumatoid arthritis, hallux abductus valgus and secondary metatarsalgia due to the increase in soles pressures [36-38]. To date, there is no strong evidence regarding the superiority of using custom-made insoles compared with prefabricated ones in patients affected by metatarsalgia [8]. In our study, the use of custom-made foot orthoses was associated with decrease in pain and disability in patients with metatarsalgia, as shown by the significant reduction of the VAS and FFI scores after treatment, but this result should be supported by randomized controlled trials to be confirmed.

In the literature, several different tools and instruments are used to evaluate the results of a conservative treatment in patients affected by metatarsalgia. Postema et al. used plantar pressure measurements as outcome markers after orthotics use [35]. Mejjad et al. used gait analysis to evaluate the results after one month of foot orthotics use [39]. Ultrasound scan is now considered, together with standard radiograph, as a first-step examination for forefoot disorders [23, 40, 41], although its use to monitor patient's outcome after therapy has not been validated yet. To our knowledge, this is the first study focused on the description of ultrasound features before and after conservative treatment of forefoot pain. After three months of foot orthotics treatment, we observed a statistically significant reduction in the number of intermetatarsal bursitis detected by ultrasound. Intermetatarsal bursitis often determines metatarsalgia, especially during walking, tingling and numbness, also mimicking MN. Small fluid collections with maximum transverse diameter of $3 \mathrm{~mm}$ in the intermetatarsal space are considered physiologic in MRI [42], although intermetatarsal bursa is not visible with ultrasound in normal conditions [27]. In pathologic conditions, intermetatarsal bursae become cystic-like lesions that can show synovium thickening and present as compressible hypoechoic structure on dynamic ultrasound, which differentiates it from a MN that is not compressible [29]. In our study, intermetatarsal bursitis was the most frequent ultrasound feature, besides being the only one presenting significant reduction after 3 months of custom-made foot orthoses. It should be noted that the frequency of intermetatarsal bursitis $(81 \%)$ was quite higher than that obtained by Iagnocco et al. [30] in 2001, where the authors found this ultrasound feature in $23 \%$ of patients with metatarsalgia. This relevant difference might have several explanations. First, we can postulate that advancements in ultrasound technology [43] could have helped us to identify small fluid effusion in the intermetatarsal spaces, as well as to differentiate them from neuromas. Then, the small sample size may have affected these data, since Iagnocco et al. evaluated 112 patients with metatarsalgia. Further, the authors did not report the experience of the operators who performed ultrasound examination that should be taken into account in this setting, since small intermetatarsal collections may be missed by less experienced operators. Our results might turn the spotlight on the role of the expansion of the intermetatarsal bursae as possible primary pain generators in patients with forefoot pain. The association of intermetatarsal bursitis and forefoot pain has been scarcely reported in the literature and no previous papers have discussed the association of disappearance/ reduction of intermetatarsal bursitis and clinical response to conservative treatment of metatarsalgia. To date, the crucial role of intermetatarsal bursitis has been postulated only in rheumatological disorders of the foot rather than in biomechanical forefoot disorders [44]. Thus, these results need to be confirmed by randomized controlled studies and could open non-negligible diagnostic and therapeutic possibilities (e.g. infiltrative treatment of intermetatarsal bursitis).

On the other hand, a statistically significant reduction of adventitious submetatarsal bursitis, joint effusion, and oedema of anterior plantar fat pad was not observed. This could be also related to the relatively small sample size. Submetatarsal adventitious bursae, as opposed to intermetatarsal ones, have no walls with mesothelial tissue [45]. Submetatarsal bursitis is often observed in the anterior plantar fat pad at the sites of higher pressure and friction, especially at the level of the first and fifth metatarsal head, presenting at ultrasound as a compressible hypoechoic structure ovoid in shape within an oedematous fat pad [29.46]. On the other hand, no improvement of the number of flexor tendinitis/ tenosynovitis and MN was observed. Probably, flexor tendinopathy plays a minor role in forefoot pain if compared with other potential pain generators. Moreover, we should 
underline that not all $\mathrm{MN}$ are symptomatic, indeed those larger in size [42] and with plantar extension [47] tend to be more symptomatic. This could explain why those patients with $\mathrm{MN}$ had pain relief despite the obvious persistence of MN itself after conservative treatment. Notably, it should be considered that $\mathrm{MN}$ can still be space-occupying, while its symptoms subside by decreasing oedema around the neuroma. A possible explanation for the discrepancy between clinical results and ultrasound findings after treatment may be the early timepoint of the second ultrasound examination, carried out only three months after the use of the orthoses, a time that could have been insufficient to determine the sonographic reduction of inflammation.

The present study has some limitations that should be pointed out. First, as already mentioned, the relatively small sample size, therefore, the generalization of our results to routinely encountered patients during clinical practice needs to be confirmed in a larger series of patients. Second, longer follow-up could help to understand how ultrasound features change over time after conservative treatment. The short time of follow-up can explain the difference between clinical benefit after orthotic therapy and the ultrasound appearance at 3-months examination. Further studies with longer follow-up are needed to confirm our hypothesis. Then, we did not include healthy subjects as controls and we did not randomize our patients in treatment arms, thus prospective randomized controlled studies should be done to understand how ultrasound features change from responding to non-responding patients. Last, we had no surgical and histopathological proof of the intermetatarsal masses ( $\mathrm{MN}$ and intermetatarsal bursitis) described by ultrasound, which was performed by only one experienced radiologist, thus no comparison was done with a second operator.

In conclusion, we have reported the ultrasound features that can be observed in patients with metatarsalgia before and after conservative treatment. Ultrasound might be able to detect some imaging features associated with the response of forefoot pain to custom-made foot orthoses, especially identifying sonographic changes of intermetatarsal bursitis, while no changes of $\mathrm{MN}$ and flexor tendinitis/tenosynovitis seem to occur in responding patients after conservative treatment. This study might help to correctly describe the ultrasound picture of patients suffering from metatarsalgia.

Funding Open access funding provided by Università degli Studi di Palermo within the CRUI-CARE Agreement. This study did not receive any funding.

\section{Declarations}

Conflict of interest The authors declare that they have no conflict of interest.
Ethical approval This study was approved by our Institutional Review Board with a waiver for patients' informed consent. All procedures performed in studies involving human participants were in accordance with the ethical standards of the institutional and/or national research committee and with the 1964 Helsinki declaration and its later amendments or comparable ethical standards.

Informed consent This study was approved by our Institutional Review Board with a waiver for patients' informed consent.

Open Access This article is licensed under a Creative Commons Attribution 4.0 International License, which permits use, sharing, adaptation, distribution and reproduction in any medium or format, as long as you give appropriate credit to the original author(s) and the source, provide a link to the Creative Commons licence, and indicate if changes were made. The images or other third party material in this article are included in the article's Creative Commons licence, unless indicated otherwise in a credit line to the material. If material is not included in the article's Creative Commons licence and your intended use is not permitted by statutory regulation or exceeds the permitted use, you will need to obtain permission directly from the copyright holder. To view a copy of this licence, visit http://creativecommons.org/licenses/by/4.0/.

\section{References}

1. Dockery G (2000) Evaluation and treatment of metatarsalgia and keratotic disorders. In: Myerson M (ed) Foot and ankle disorders. Philadelphia:W.B. Saunders Co, pp 359-377

2. DiPreta JA (2014) Metatarsalgia, lesser toe deformities, and associated disorders of the forefoot. Med Clin North Am 98:233-251. https://doi.org/10.1016/j.mcna.2013.10.003

3. Leone A, Vitiello C, Gullì C et al (2020) Bone and soft tissue infections in patients with diabetic foot. Radiol Med 12:177-187. https://doi.org/10.1007/s11547-019-01096-8

4. Chianca V, Orlandi D, Messina C et al (2019) Interventional therapeutic procedures to treat degenerative and inflammatory musculoskeletal conditions: state of the art. Radiol Med 124:1112-1120. https://doi.org/10.1007/s11547-019-01018-8

5. Silvestri E, Barile A, Albano D et al (2018) Interventional therapeutic procedures in the musculoskeletal system: an Italian Survey by the Italian College of Musculoskeletal Radiology. Radiol Med 123:314-321. https://doi.org/10.1007/s11547-017-0842-7

6. Espinosa N, Brodsky JW, Maceira E (2010) Metatarsalgia. J Am Acad Orthop Surg 18:474-485. https://doi.org/10.5435/00124 635-201008000-00004

7. Nawoczenski DA, Janisse DJ (2004) Foot orthoses in rehabilitation-What's new. Clin Sports Med 23:157-167. https://doi.org/ 10.1016/S0278-5919(03)00087-5

8. Lucas-Cuevas AG, Perez-Soriano P, Llana-Belloch S et al (2014) Effect of custom-made and prefabricated insoles on plantar loading parameters during running with and without fatigue. J Sports Sci 32:1712-1721. https://doi.org/10.1080/02640414.2014. 915422

9. Naraghi R, Bremner A, Slack-Smith L et al (2017) Radiographic analysis of feet with and without Morton's neuroma. Foot Ankle Int. 38:310-317. https://doi.org/10.1177/1071100716674998

10. Sconfienza LM, Orlandi D, Lacelli F et al (2015) Dynamic highresolution US of ankle and midfoot ligaments: normal anatomic structure and imaging technique. Radiographics 35:164-178. https://doi.org/10.1148/rg.351130139

11. Albano D, Martinelli N, Bianchi A et al (2017) Clinical and imaging outcome of osteochondral lesions of the talus treated using 
autologous matrix-induced chondrogenesis technique with a biomimetic scaffold. BMC Musculoskelet Disord 18:306. https://doi. org/10.1186/s12891-017-1679-x

12. Bellelli A, Silvestri E, Barile A et al (2019) Position paper on magnetic resonance imaging protocols in the musculoskeletal system (excluding the spine) by the Italian College of Musculoskeletal Radiology. Radiol Med 124:522-538. https://doi.org/10. 1007/s11547-019-00992-3

13. Albano D, Martinelli N, Bianchi A et al (2018) Posterior tibial tendon dysfunction: Clinical and magnetic resonance imaging findings having histology as reference standard. Eur J Radiol 99:55-61. https://doi.org/10.1016/j.ejrad.2017.12.005

14. Szaro P, Polaczek M, Świątkowski J et al (2020) How to increase the accuracy of the diagnosis of the accessory bone of the foot? Radiol Med 125:188-196. https://doi.org/10.1007/ s11547-019-01104-x

15. Foti G, Catania M, Caia S et al (2019) Identification of bone marrow edema of the ankle: diagnostic accuracy of dual-energy CT in comparison with MRI. Radiol Med 124:1028-1036. https://doi. org/10.1007/s11547-019-01062-4

16. Bruno F, Arrigoni F, Palumbo P et al (2019) New advances in MRI diagnosis of degenerative osteoarthropathy of the peripheral joints. Radiol Med 124:1121-1127. https://doi.org/10.1007/ s11547-019-01003-1

17. Albano D, Cortese MC, Duarte A et al (2020) Predictive role of ankle MRI for tendon graft choice and surgical reconstruction. Radiol Med 125:763-769. https://doi.org/10.1007/ s11547-020-01177-z

18. Salaffi F, Carotti M, Barile A (2019) Musculoskeletal imaging of the inflammatory and degenerative joints: current status and perspectives. Radiol Med 124:1067-1070. https://doi.org/10.1007/ s11547-019-01004-0

19. Hodes A, Umans H (2018) Metatarsalgia. Radiol Clin North Am 56:877-892. https://doi.org/10.1016/j.rcl.2018.06.004

20. Albano D, Messina C, Usuelli FG et al (2017) Magnetic resonance and ultrasound in achilles tendinopathy: predictive role and response assessment to platelet-rich plasma and adipose-derived stromal vascular fraction injection. Eur J Radiol 95:130-135. https://doi.org/10.1016/j.ejrad.2017.08.006

21. Okano T, Mamoto K, Di Carlo M et al (2019) Clinical utility and potential of ultrasound in osteoarthritis. Radiol Med 124:11011111. https://doi.org/10.1007/s11547-019-01013-z

22. Gitto S, Messina C, Chianca V et al (2020) Superb microvascular imaging (SMI) in the evaluation of musculoskeletal disorders: a systematic review. Radiol Med 125:481-490. https://doi.org/10. 1007/s11547-020-01141-x

23. Sconfienza LM, Albano D, Allen G et al (2018) Clinical indications for musculoskeletal ultrasound updated in 2017 by European society of musculoskeletal radiology (ESSR) consensus. Eur Radiol 28:5338-5351. https://doi.org/10.1007/s00330-018-5474-3

24. Klauser AS, Tagliafico A, Allen GM et al (2012) Clinical indications for musculoskeletal ultrasound: a delphi-based consensus paper of the European society of musculoskeletal radiology. Eur Radiol 22:1140-1148. https://doi.org/10.1007/s00330-018-5474-3

25. Williamson A, Hoggart B (2005) Pain: a review of three commonly used pain rating scales. J Clin Nurs 14:798-804. https:// doi.org/10.1111/j.1365-2702.2005.01121.x

26. Budiman-Mak E, Conrad KJ, Roach KE (1991) The Foot Function Index: a measure of foot pain and disability. J Clin Epidemiol 44:561-570. https://doi.org/10.1016/0895-4356(91)90220-4

27. Koski JM (1998) Ultrasound detection of plantar bursitis of the forefoot in patients with early rheumatoid arthritis. J Rheumatol 25:229-230

28. Ashman CJ, Klecker RJ, Yu JS (2001) Forefoot pain involving the metatarsal region: differential diagnosis with MR imaging.
Radiographics 21:1425-1440. https://doi.org/10.1148/radiograph ics.21.6.g01nv071425

29. Ganguly A, Warner J, Aniq H (2018) Central metatarsalgia and walking on pebbles: beyond morton neuroma. AJR Am J Roentgenol 210:821-833. https://doi.org/10.2214/AJR.17.18460

30. Iagnocco A, Coari G, Palombi G et al (2001) Sonography in the study of metatarsalgia. J Rheumatol 28:1338-1340

31. Torriani M, Kattapuram SV (2003) Technical innovation. Dynamic sonography of the forefoot: the sonographic Mulder sign. AJR Am J Roentgenol. 180:1121-1123. https://doi.org/10. 2214/ajr.180.4.1801121

32. Draghi F, Gitto S, Bortolotto $C$ et al (2017) Imaging of plantar fascia disorders: findings on plain radiography, ultrasound and magnetic resonance imaging. Insights Imaging 8:69-78. https:// doi.org/10.1007/s13244-016-0533-2

33. Di Leo G, Sardanelli F (2020) Statistical significance: $p$ value, 0.05 threshold, and applications to radiomics-reasons for a conservative approach. Eur Radiol Exp. 4:18. https://doi.org/10.1186/ s41747-020-0145-y

34. Menz HB (2015) Biomechanics of the ageing foot and ankle: a mini-review. Gerontology 61:381-388. https://doi.org/10.1159/ 000368357

35. Postema K, Burm PET, Zande MEVD et al (1998) Primary metatarsalgia: the influence of a custom moulded insole and a rockerbar on plantar pressure. Prosthet Orthot Int 22:35-44. https://doi. org/10.3109/03093649809164455

36. Arias-Martín I, Reina-Bueno M, Munuera-Martínez PV (2018) Effectiveness of custom-made foot orthoses for treating forefoot pain: a systematic review. Int Orthop 42:1865-1875. https://doi. org/10.1007/s00264-018-3817-y

37. Jiunn-Horng K, Min-Der C, Shih-Ching C et al (2006) Correlations between subjective treatment responses and plantar pressure parameters of metatarsal pad treatment in metatarsalgia patients: a prospective study. BMC Musculoskelet Disord 7:95. https://doi. org/10.1186/1471-2474-7-95

38. Sperryn PN, Restan L (1983) Podiatry and the sports physician-an evaluation of orthoses. Br J Sports Med 17:129-134. https://doi. org/10.1136/bjsm.17.4.129

39. Mejjad O, Vittecoq O, Pouplin S et al (2004) Foot orthotics decrease pain but do not improve gait in rheumatoid arthritis patients. Jt Bone Spine 71:542-545. https://doi.org/10.1016/j. jbspin.2003.09.007

40. Bianchi S (2014) Practical US of the foot. J Ultrasound 17:151164. https://doi.org/10.1007/s40477-014-0078-5

41. Ansede G, Lee JC, Healy JC (2010) Musculoskeletal sonography of the normal foot. Skeletal Radiol 39:225-242. https://doi.org/ 10.1007/s00256-009-0697-7

42. Zanetti M, Strehle JK, Zollinger H et al (1997) Morton neuroma and fluid in the intermetatarsal bursae on MR images of 70 asymptomatic volunteers. Radiology 203:516-520. https://doi.org/10. 1148/radiology.203.2.9114115

43. Albano D, Aringhieri G, Messina C, De Flaviis L, Sconfienza LM (2020) High-frequency and ultra-high frequency ultrasound: musculoskeletal imaging up to $70 \mathrm{MHz}$. Semin Musculoskelet Radiol 24:125-134. https://doi.org/10.1055/s-0039-3401042

44. Albtoush OM, Xenitidis T, Horger M (2019) Intermetatarsal bursitis as first disease manifestation in different rheumatological disorders and related MR-imaging findings. Rheumatol Int 39:2129-2136. https://doi.org/10.1007/s00296-019-04381-x

45. Bowen CJ, Dewbury K, Sampson M et al (2008) Musculoskeletal ultrasound imaging of the plantar forefoot in patients with rheumatoid arthritis: inter-observer agreement between a podiatrist and a radiologist. J Foot Ankle Res 1:5. https://doi.org/10.1186/ 1757-1146-1-5

46. Resnick DR (1995) Diagnosis of bone and joint disorders, 3rd edition. p 668 
47. Bencardino J, Rosenberg ZS, Beltran J et al (2000) Morton's neuroma: is it always symptomatic? AJR Am J Roentgenol 175:649653. https://doi.org/10.2214/ajr.175.3.1750649
Publisher's Note Springer Nature remains neutral with regard to jurisdictional claims in published maps and institutional affiliations. 